\title{
Anuria as a Chief Presentation of Takayasu Arteritis
}

\author{
Sara K. Alrasheed ${ }^{1}$, Basel Alheijani ${ }^{1}$, Rahmah Alzahrani' ${ }^{1}$, Shaker Alshehri ${ }^{2}$ \\ ${ }^{1}$ Department of Medicine, King Abdulaziz Medical City, Ministry of National Guard-Health Affairs, Riyadh, KSA \\ ${ }^{2}$ Department of Radiology, King Abdulaziz Medical City, Ministry of National Guard-Health Affairs, Riyadh, KSA \\ Email: drsara555@gmail.com
}

How to cite this paper: Alrasheed, S.K., Alheijani, B., Alzahrani, R. and Alshehri, S. (2021) Anuria as a Chief Presentation of Takayasu Arteritis. Case Reports in Clinical Medicine, 10, 24-33.

https://doi.org/10.4236/crcm.2021.102004

Received: December 10, 2020

Accepted: February 20, 2021

Published: February 23, 2021

Copyright (c) 2021 by author(s) and Scientific Research Publishing Inc. This work is licensed under the Creative Commons Attribution International License (CC BY 4.0).

http://creativecommons.org/licenses/by/4.0/

(c) (i) Open Access

\begin{abstract}
Takayasu vasculitis is a rare type of large vessel vasculitis that primarily affects the aorta and its main branches; signs and symptoms are usually due to systemic inflammation or ischemia of an organ or a limb, depending on the group of blood vessels involved. In addition, Takayasu arteritis is associated with increased platelet and coagulation activity, leading to a hypercoagulable state and thrombus formation. We report a case of a 47 -year-old male who presented with a history of complete anuria for 3 days and was found to have progressively worsening kidney function. Renal Doppler ultrasound confirmed the presence of bilateral renal artery thrombosis, while contrast-enhanced computed tomography (CECT) of the abdomen and pelvis showed extensive abdominal aortic thrombosis with radiological findings consistent with large vessel vacuities. After catheter-directed thrombolytic therapy of the renal arteries, the patient started producing urine and his kidney function significantly improved. Later, positron emission tomography scan (PET) confirmed large vessel Takayasu arteritis. Echocardiography showed no intracardiac thrombus, along with an extensive work-up for thrombophilia, as autoimmune and vasculitis serology came back negative. This is an extremely rare presentation of Takayasu arteritis, with an unusual recovery of acute renal failure after prolonged anuria due to bilateral renal artery thrombosis.
\end{abstract}

\section{Keywords}

Takayasu, Anuria, Vasculitis

\section{Background}

Bilateral renal artery thrombosis, secondary to aortic thrombosis is infrequent, often associated with atherosclerotic and/or aneurysmal changes, rarely caused 
by other conditions. Takayasu large vessel vasculitis (nonspecific) is one of the rare causes of aortic thrombosis [1], and is often missed, although it is a reversible and treatable condition.

Takayasu vasculitis is a rare type of granulomatous vessel vasculitis, and it is considered a chronic inflammatory condition that primarily affects the aorta and its main branches; the exact cause is not well established. Clinical presentation is highly variable, depending on the territory of vascular involvement [1]. In addition, Takayasu arteritis (TA) is associated with increased platelet and coagulation activity, leading to a hypercoagulable state and thrombus formation [2].

This case report describes a rare case of TA presenting with anuric renal failure. Written informed patient consent was obtained in the present study.

\section{Case Presentation}

A 47-year-old male presented with an abrupt onset of anuria for 3 days. There was no history of flank pain, dysuria, hematuria, fever, vomiting, or diarrhea. Ten days prior to his presentation, he was complaining of abdominal pain, mainly around the umbilicus, associated with bloating and constipation. $\mathrm{He}$ went to another hospital and was managed with: bisacodyl, oral lactulose, and fleet enema, and was discharged. Review of other systems revealed a history of general fatigue and lower limb claudication after a walking distance of 20 to 30 meters. There was no history of skin rashes, joint pain or swelling, cough, chest pain, palpitation, or shortness of breath. Moreover, there was no history of headache, or any neurological symptoms. His past medical history was significant for hypertension and lumbar spine disk prolapse.

Past medical history was significant for a hospitalization 5 years ago, for unprovoked massive bilateral pulmonary embolism. Investigations at that admission included: thrombophilia work-up, which was non-revealing, an echocardiography which showed reduced left ventricular ejection fraction (EF) of $45 \%$. Follow-up computerized tomography (CT) of the coronary arteries revealed a zero calcium score and a mild nonobstructive coronary artery disease (CAD) in the proximal left anterior descending coronary artery (LAD). Cardiac MRI showed an old infarction in the apical segment, with no left ventricular (LV) cavity thrombus. At the time, there was no history of thrombosis (before that presentation), abdominal aortic aneurysm, or renal stones in the patient's history or family. His medications included aspirin, valsartan, metoprolol, and atorvastatin. He did not smoke, use alcohol, or illicit drugs.

Physical exam at presentation: patient was conscious, oriented, not in pain or distress, body temperature of $36.3^{\circ} \mathrm{C}$, blood pressure: left arm $127 / 80$, right arm $121 / 78$, left leg 57/30, right leg 62/31, heart rate of 79 beats per minute, regular with absent left radial pulse, lower limb pulse was palpable but weak bilaterally, respiratory rate of 21 breaths per minute and pulsoximetry of $98 \%$ on room air. Cardiovascular examinations revealed normal heart sounds, no murmurs, normal JVP, no carotid bruit, and no pedal edema. His abdominal exam revealed mild tenderness to palpation, more on the right flank compared to the left: no 
rebound, guarding, distention, or organomegaly. The rest of the exam was normal.

\subsection{Investigations If Relevant}

The initial emergency department evaluation included an electrocardiogram (EKG), which was unremarkable, a white blood cell (WBC) of $7.10 \mathrm{k} / \mathrm{uL}$ (normal range 4 -11), a hemoglobin of $136 \mathrm{~g} / \mathrm{dL}$ (normal range 135 - $180 \mathrm{gm} / \mathrm{L}$ ), a platelet count of $302 \mathrm{~K} / \mathrm{uL}$ (normal range 150 - 400). Other laboratory investigations revealed a creatinine of $500 \mathrm{umol} / \mathrm{L}$ (normal range $64-110 \mathrm{umol} / \mathrm{L}$ ), while his last documented baseline creatinine was $70 \mathrm{umol} / \mathrm{L}$, a blood urea nitrogen (BUN) of 8 (normal range $7.4-3.2 \mathrm{mmol} / \mathrm{L}$ ), a potassium of 3.4 (normal range 3.5 - $5.1 \mathrm{mmol} / \mathrm{L}$ ), a bicarbonate level of 19 (normal range 22 - $29 \mathrm{mmol} / \mathrm{L}$ ). Furthermore, his lactate dehydrogenase (LDH) level was 840 (normal range 125 - 220), and erythrocyte sedimentation rate (ESR) was $49 \mathrm{~mm} / \mathrm{H}$ (normal range 0 - $15 \mathrm{~mm} / \mathrm{hr}$ ). Other laboratory work-up included liver profile, lactic acid level, and coagulation profile which were within normal limits. Patient was not able to produce urine even after Foley's catheter insertion. Non-enhanced CT scan of the abdomen and pelvis showed no overt obstructive uropathy or renal stones. On admission, his creatinine level increased to $900 \mathrm{umol} / \mathrm{L}$. Renal doppler ultrasound was done and showed findings suggestive of evolving bilateral main renal artery thrombosis versus stenosis (Figure 1).

Further assessment by contrast-enhanced CT scan (CECT) of the abdomen and pelvis revealed thrombosis of the renal arteries, the infra-renal abdominal aorta, distal splenic artery, superior and inferior mesenteric arteries, along with bilateral scattered areas of renal and splenic hypoperfusion. There was chronic total occlusion of the common iliac arteries, bilaterally. The thrombosed vessels showed mural thickening with progressive enhancement at delayed phases (Figure 2).

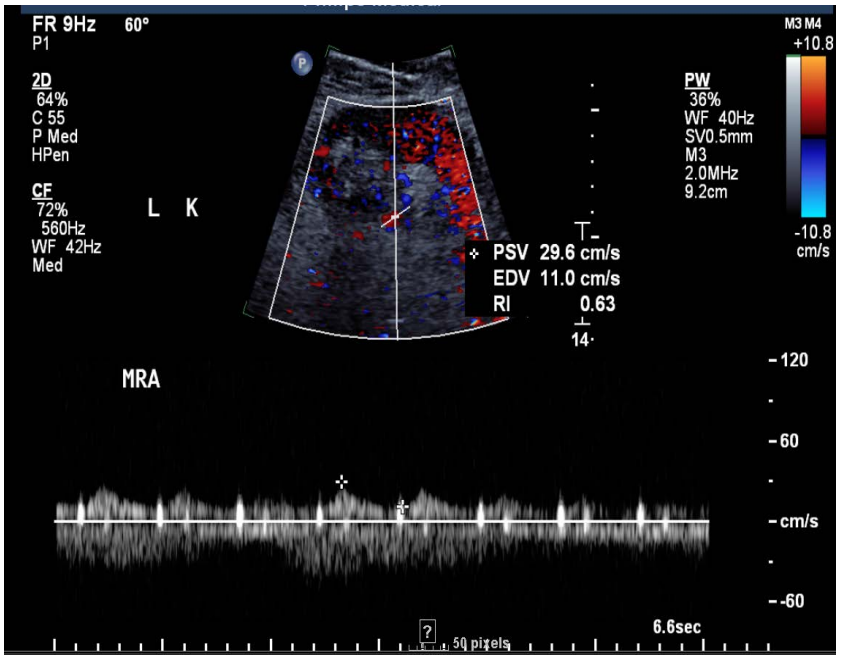

(a)

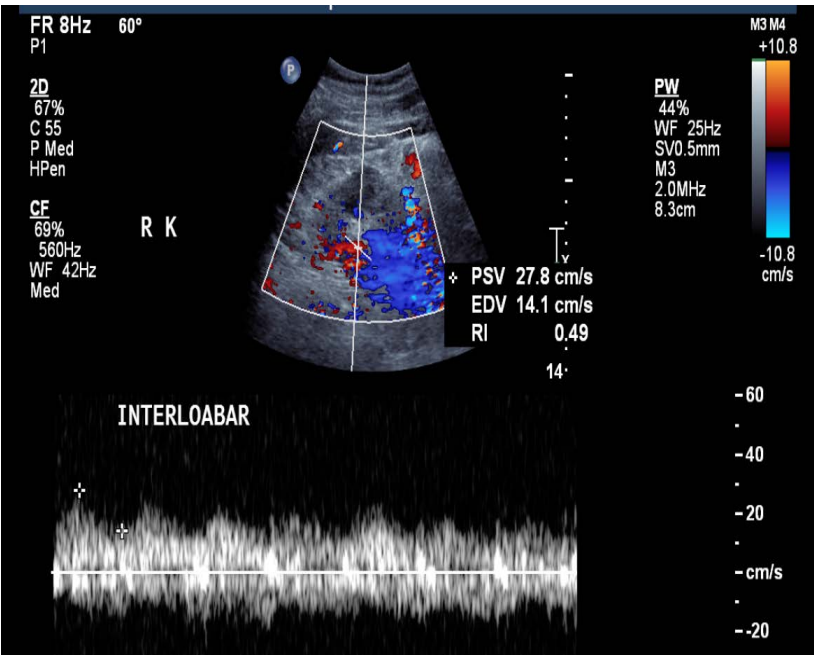

(b)

Figure 1. (a) Sagittal color Doppler ultrasound of the right kidney (a) interlobar segment shows significant drop in the peak velocity and tardus parvus wave from in addition to low resistive index. (b) Left kidney color Doppler of the main renal artery showed low peak velocity and dampening. 

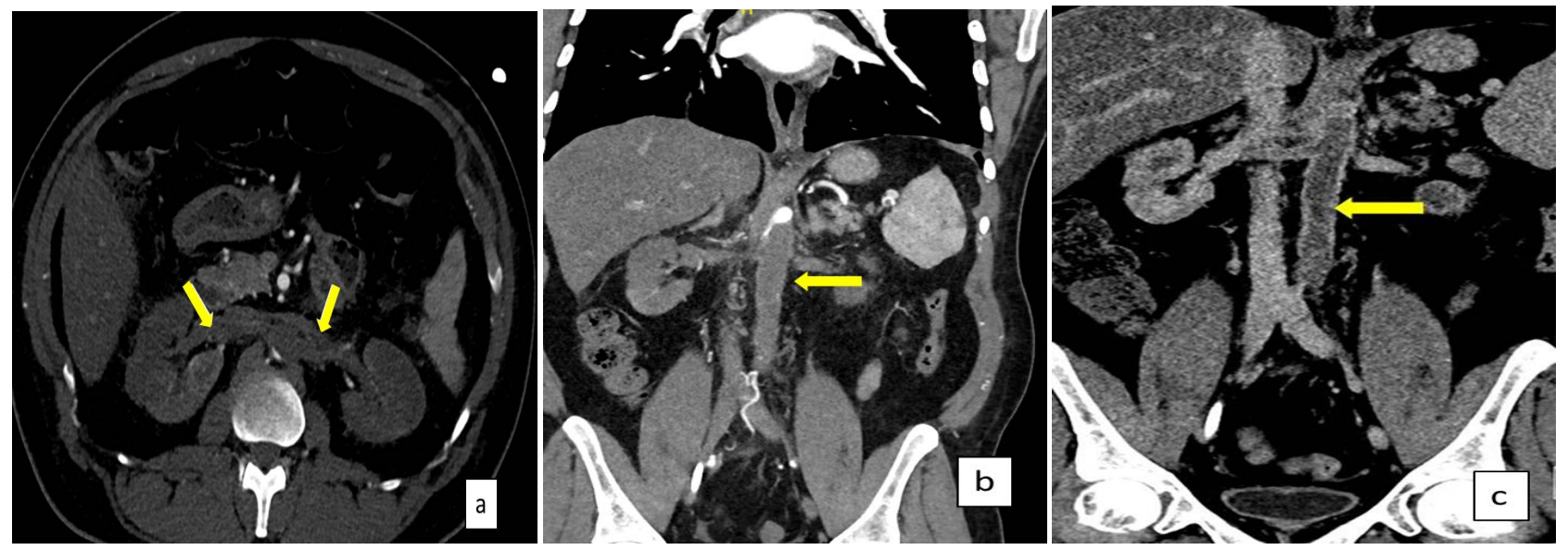

Figure 2. Axial (a) CECT image shows thrombosis of the renal arteries bilaterally (arrow), and coronal (b) image shows infra renal abdominal aorta (arrow) thrombosis extending to the iliac branches. (c) Selected coronal CT shows mural thickening of the infra-renal abdominal aorta and renal artery with delayed enhancement of the wall.

An urgent interventional radiology procedure was carried out utilizing the left brachial and radial arterial accesses, the angiogram revealed total aortoiliac occlusion, bilateral renal arteries, and origin of the superior mesenteric arteries thrombosis (Figure 3).

PET scan showed mild symmetrical increased activity seen in both subclavian arteries of both upper arms and femoral arteries suggestive of vasculitis.

Echocardiography revealed normal left ventricle size, ejection fraction of 45 to $50 \%$, normal valves, and no overt intracardiac thrombus detected.

Rheumatological workup included: antinuclear antibodies (ANA), anti-double stranded DNA antibody (anti-dsDNA) (ELISA), anticardiolipin IgG, anticardiolipin IgA, anti- $\beta 2$ glycoprotein IgA, anti- $\beta 2$ glycoprotein IgG, anti- $\beta 2$ glycoprotein IgM, anti-glomerular basement membrane antibody (anti-GBM), anti-neutrophil cytoplasmic antibodies (ANCAs), and perinuclear anti-neutrophil cytoplasmic antibodies (P-ANCA), which were all within normal range.

Hematological workup, such as gene testing for JAK 2 mutation and prothrombin mutation, factor $\mathrm{V}$ leiden was negative. Paroxysmal nocturnal hemoglobinuria (PNH) work-up was negative. Antiphospholipid antibody work-up was repeated and came back negative again.

\subsection{Differential Diagnosis If Relevant}

Important potential diagnoses of a patient presenting with bilateral renal artery thrombosis include; cardiac emboli secondary to atrial fibrillation or endocarditis, atheroemboli, large and medium vessel vasculitis, lupus vasculitis, sickle cell disease, fibromuscular dysplasia of the renal arteries, dissecting aneurysms of the aorta, and antiphospholipid antibody syndrome.

In this patient, there was no previous past medical history of sickle cell disease, angioplasty, angiography, or cardiovascular surgery. No evidence of atrial fibrillation via ECG after reviewing all ECGs. Echo showed no vegetation and his blood cultures were negative. Initial unenhanced CT of his abdomen and pelvis 

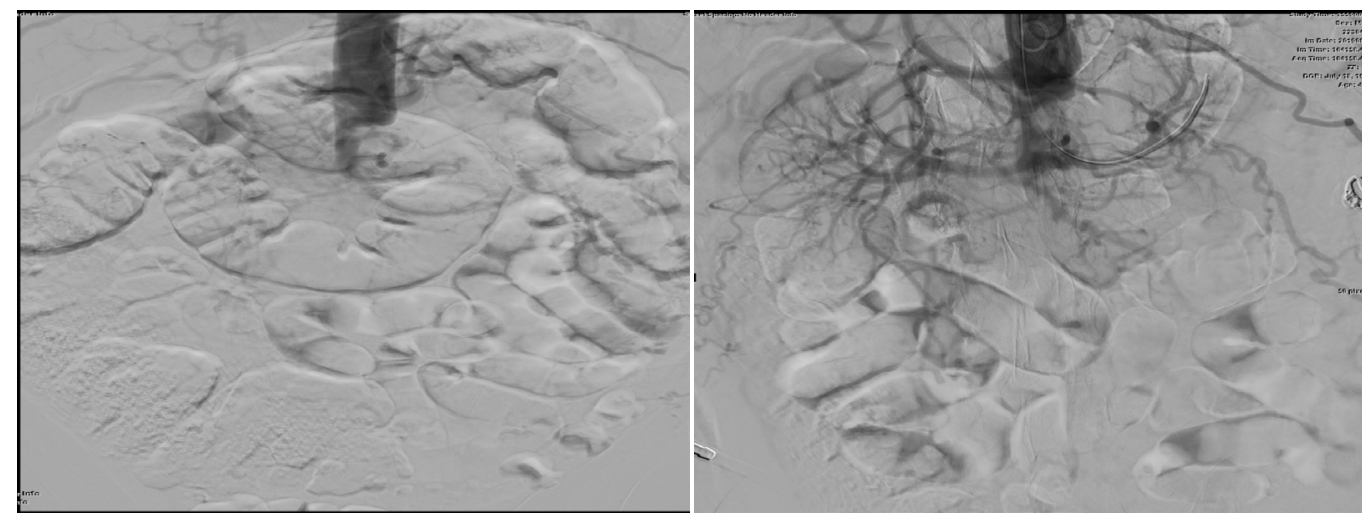

Figure 3. Selective DSA image for the abdominal aortogram from the brachial artery access show occlusion of the infrarenal aorta, renal arteries and revealed total aortoiliac occlusion, bilateral renal arteries, and origin of the superior mesenteric arteries thrombosis.

showed no stones or hydronephrosis. Renal Doppler US and CECT of his abdomen and pelvis confirmed the presence of aortic thrombosis extending to both renal arteries. Further hematological work-up needed in such a young patient included lupus antibodies, JAK-2 mutation, antiphospholipid antibodies, factor V Leiden, and prothrombin mutation, which were all unremarkable in our patient. Besides that, large vessels' vasculitis was part of the differential, so that PET scan was helpful in this case to confirm the diagnosis.

\subsection{Treatment If Relevant}

Catheter-directed thrombolysis was done by interventional radiology through radial and brachial artery for bilateral renal artery thrombosis (Figure 4). Anticoagulation with intravenous heparin, then warfarin $3 \mathrm{mg}$ once daily with an international normalized ratio (INR) target of 2 - 3 indefinitely. Corticosteroids, specifically prednisolone, was started with a dose of $25 \mathrm{mg}$ per day, tapered down weekly by $5 \mathrm{mg}$ to reach a dose of $5 \mathrm{mg}$ daily. Methotrexate was initiated as well, with a dose of $10 \mathrm{mg}$ once per week.

\section{Outcome and Follow-Up}

Aortogram, the day after thrombolytic therapy, showed resumption of the flow to the renal artery bilaterally, and follow-up renal Doppler ultrasound confirmed no thrombus in renal arteries (Figure 4). The patient initially received 3 sessions of hemodialysis in the first few days of admission; however, after thrombolysis, he started to produce good urine output (around 3 liters per day).

After 4 months follow-up, his creatinine level was 113 umol/L (reached 1075 um/L upon admission), and he was passing good amount of urine with no overt new organ involvement.

\section{Discussion Include a Very Brief Review of Similar Published Cases}

Takayasu arteritis (TA) is a chronic granulomatous pan arteritis, predominantly 

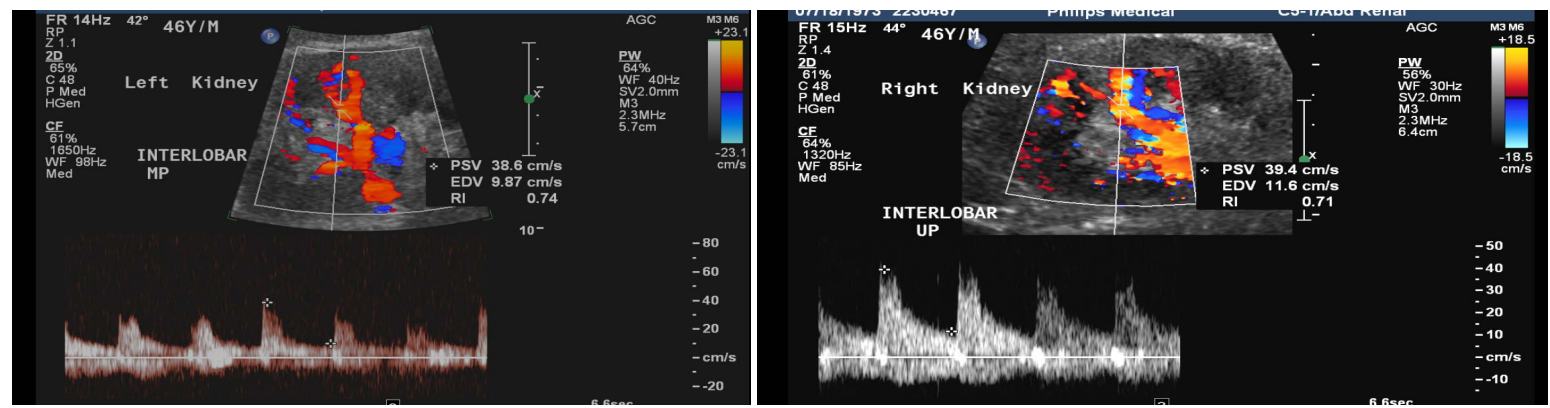

Figure 4. Selective DSA images of the renal artery after 12 hours of catheter directed thrombolysis shows patency of the renal arteries bilaterally with resumption of the flow to the renal artery bilaterally.

affecting females, but males are also affected [3]. The American Rheumatological Society considers three of the following six criteria necessary for a definite diagnosis of Takayasu's disease: age of onset $<40$, claudication of the limbs, decreased pulse of the brachial artery, difference in systolic blood pressure $>10$ mmHg between the two arms, murmurs of the subclavian arteries or the aorta, and abnormal arteriography [4]. Our patient exhibited three of the six criteria; decrease pulse of brachial artery, lower limb claudication and abnormal arteriography, with unknown age of onset and late diagnosis.

The clinical manifestations of TA are varied, and can be asymptomatic for several years, or can present as nonspecific systemic symptoms [1]. In this case, diagnosis of TA was delayed due to the nonspecific nature of the symptoms upon presentation with absent urination.

The course of the disease can be divided into an early active inflammatory phase, and a late chronic phase. The active phase involves fever, malaise, night sweats, loss of appetite, weight loss, headaches, dizziness, arthralgia, skin rashes, and rarely acute vascular events. The late chronic phase is the result of arterial stenosis and/or occlusion, which primarily involves the aorta and its main branches, and the coronary and pulmonary arteries [1].

Among patients with TA, the prevalence of renal artery involvement was more common in Chinese patients compared to those in North America, Northern Europe, and Africa with renal artery stenosis and occlusion being the most common abnormalities [5] [6]. In previous retrospective analysis of 411 patients diagnosed with Takayasu's arteritis in a medical center in china, 201 diagnosed with renal involvements, $78 \%$ among those have renal artery stenosis [4]. We reviewed 14 case reports and case series that included a total of $22 \mathrm{pa}-$ tients with adult onset renovascular TA (Table 1), $81.8 \%$ of them presented with hypertension [7]-[17]. Moreover, 2 of these reported cases primarily presented with anuria, however, those patients had solitary kidneys [18] [19], and only one case presented with anuria secondary to bilateral renal artery occlusion [20] which was similar to our case who mainly presented with anuric renal failure due to extensive aortic thrombosis with bilateral renal artery involvements.

The angiographic classification of TA includes six findings: I: branches of aortic arch, IIa: ascending aorta, aortic arch and its branches, IIb: ascending aorta, 
Table 1. Summary of data derived from the literature review.

\begin{tabular}{|c|c|c|c|c|}
\hline Author & Year & Gender and Age & Clinical presentation & Radiological findings \\
\hline Fadouach et al. [7] & $\begin{array}{c}\text { Case series of } 8 \\
\text { patients between } \\
1983-1995\end{array}$ & Case series of 8 patient & Hypertension & Unilateral or bilateral renal artery stenosis. \\
\hline Dardik et al. [8] & 1997 & 19-year-old female & Malignant hypertension & $\begin{array}{l}\text { Severe proximal stenosis of the renal, celiac, and } \\
\text { superior mesenteric arteries, and a thickened } \\
\text { abdominal aorta. }\end{array}$ \\
\hline Wolak et al. [9] & 2003 & 20-year-old female & Malignant hypertension & $\begin{array}{l}\text { Narrowing of the left subclavian artery with } \\
\text { collateral flow, unusually well-developed intercostal } \\
\text { arteries, narrowing of the aorta from the superior } \\
\text { mesenteric artery to the inferior mesenteric artery, } \\
\text { and tight bilateral renal artery stenosis. }\end{array}$ \\
\hline Zumrutdal et al. [10] & 2005 & 28-year-old female & Hypertensive urgency & $\begin{array}{l}\text { Multiple severe stenoses or occlusions of the } \\
\text { branches of the abdominal aorta and arcus aortae } \\
\text { together with bilateral renal artery involvement. }\end{array}$ \\
\hline Galesić et al. [11] & 2005 & 18-year-old female & hypertension & Bilateral renal artery stenosis. \\
\hline Moorthy et al. [12] & 2007 & 24-year-old female & Malignant hypertension & $\begin{array}{l}\text { Abdominal aorta documented dissection of } \\
\text { abdominal aorta arising at the level of sub } \\
\text { diaphragm extending up to the right common iliac } \\
\text { artery. It also demonstrated the intimal tear below } \\
\text { the level of renal arteries. }\end{array}$ \\
\hline Borazan et al. [13] & 2009 & 19-year-old female & Hypertensive emergency & $\begin{array}{l}\text { Stenosis of left renal artery and arterial wall } \\
\text { thickening among the aorta were detected. }\end{array}$ \\
\hline Hassani et al. [14] & 2016 & 16-year-old female & Malignant hypertension & $\begin{array}{l}\text { Occlusion of both subclavian arteries occlusion of } \\
\text { left renal artery and thickening and narrowing of } \\
\text { the sub-renal abdominal aortic wall. }\end{array}$ \\
\hline Horino et al. [15] & 2018 & 82-year-old female & $\begin{array}{l}\text { Chronic hypertension with } \\
\text { progressive renal } \\
\text { dysfunction }\end{array}$ & $\begin{array}{l}\text { Thickening and enhancement in the ascending, } \\
\text { thoracic descending, and abdominal aorta and its } \\
\text { branches, and the carotid, subclavian, and renal } \\
\text { arteries. }\end{array}$ \\
\hline Tsuchida et al. [16] & 2019 & 18-year-old female & $\begin{array}{l}\text { Hypertension, metabolic } \\
\text { alkalosis and nephrotic } \\
\text { range proteinuria }\end{array}$ & Right renal artery stenosis. \\
\hline Vargas-Hitos et al. [17] & 2019 & 32-year-old female & Malignant hypertension & $\begin{array}{l}\text { Bilateral renal artery stenosis with significant } \\
\text { abdominal aortic stenosis. }\end{array}$ \\
\hline Delles C et al. [18] & 2002 & 35-year-old female & Anuria in solitary kidney & Occlusion of the renal artery. \\
\hline Dasari et al. [19] & 2011 & 72-year-old female & $\begin{array}{l}\text { Hypertension and anuria in } \\
\text { solitary kidney }\end{array}$ & Severe renal artery stenosis. \\
\hline Yadla et al. [20] & 2017 & 30-year-old female & Hypertension and anuria & $\begin{array}{l}\text { Narrowing of the aorta below the origin of inferior } \\
\text { mesenteric artery till the bifurcation, and the renal } \\
\text { arteries could not be visualized. }\end{array}$ \\
\hline
\end{tabular}

aortic arch and its branches, and thoracic descending aorta, III: thoracic descending aorta, abdominal aorta, and/or renal arteries, IV: abdominal aorta and/or renal arteries, V: combination of Types IIb and IV [21]. Our patient was classified as TA, type IV, in which renal angiogram revealed bilateral renal arteries and origin of the superior mesenteric arteries with acute thrombosis. 
Immunosuppressants such as prednisolone and methotrexate can lead to significant improvement in TA patients; however, around $50 \%$ of patients of TA progress to a stage that requires one or more surgical procedures, such as bypass grafts, angioplasty, or stent placement. In our case, thrombosis of the abdominal aorta and renal artery were the main cause of renal artery occlusion, for that the interventional radiology team was involved, and successful catheter-directed thrombolysis was conducted.

TA is associated with favorable long-term renal and nonrenal outcomes [22]. The early initiation of therapy may result in reversible vessel pathology.

\section{Conclusion}

This case report signifies the importance of early identification of symptoms and signs of rare diseases such as Takayasu Arteritis which in turn leads to early diagnosis and treatment. This will hopefully prevent permanent end organ damage and may allow for fast recovery after sustained ischemia.

\section{Learning Points/Take Home Messages; 3 - 5 Bullet Points}

- Bilateral renal artery thrombosis is a rare cause of anuria and worsening kidney function, which needs to be diagnosed and treated when other sinister causes like obstructive uropathy are excluded.

- In addition to ischemia and stenosis, Takayasu vasculitis rarely presents with thrombosis formation, leading to significant organ damage that needs to be treated very early.

- Catheter-directed thrombolytic therapy for renal artery thrombosis results in rapid improvement in kidney function and prevents permanent severe renal damage and the need for renal replacement therapy.

\section{Conflicts of Interest}

The authors declare no conflicts of interest regarding the publication of this paper.

\section{References}

[1] Johnston, S.L., Lock, R.J. and Gompels, M.M. (2002) Takayasu Arteritis: A Review. Journal of Clinical Pathology, 55, 481-486.

[2] Emmi, G., Silvestri, E., Squatrito, D., Amedei, A., Niccolai, E., D’Elios, M.M., et al. (2015) Thrombosis in Vasculitis: From Pathogenesis to Treatment. Thrombosis Journal, 13, Article No. 15. https://doi.org/10.1186/s12959-015-0047-z

[3] Kerr, G.S., Hallahan, C.W., Giordano, J., Leavitt, R.Y., Fauci, A.S., Rottem, M. and Hoffman, G.S. (1994) Takayasu Arteritis. Annals of Internal Medicine, 120, 919-929. https://doi.org/10.7326/0003-4819-120-11-199406010-00004

[4] Calabrese, L.H., Michel, B.A., Bloch, D.A., Arend, W.P., Edworthy, S.M., Fauci, A.S., et al. (1990) The American College of Rheumatology 1990 Criteria for the Classification of Hypersensitivity Vasculitis. Arthritis \& Rheumatism, 33, 1108-1113. https://doi.org/10.1002/art.1780330808 
[5] Chen, Z., Li, J., Yang, Y., Li, H., Zhao, J., Sun, F. and Zeng, X. (2018) The Renal Artery Is Involved in Chinese Takayasu's Arteritis Patients. Kidney International, 93, 245-251. https://doi.org/10.1016/j.kint.2017.06.027

[6] Gudbrandsson, B., Molberg, Ø., Garen, T. and Palm, Ø. (2017) Prevalence, Incidence, and Disease Characteristics of Takayasu Arteritis by Ethnic Background: Data from a Large, Population-Based Cohort Resident in Southern Norway. Arthritis Care \& Research, 69, 278-285. https://doi.org/10.1002/acr.22931

[7] Delles, C., Weidner, S., Schobel, H.P. and Rupprecht, H.D. (2002) Renal-Artery Stenosis in a Patient with Takayasu's Arteritis. Nephrology Dialysis Transplantation, 17, 1339-1341. https://doi.org/10.1093/ndt/17.7.1339

[8] Dasari, T.W., Hanna, E.B. and Exaire, J.E. (2011) Resolution of Anuric Acute Kidney Injury after Left Renal Angioplasty and Stenting for a Totally Occlusive in-Stent Restenosis of a Solitary Kidney. The American Journal of the Medical Sciences, 341, 163-165. https://doi.org/10.1097/MAJ.0b013e3181fb79af

[9] Yadla, M., Khandalvelli, P. and Uppin, M. (2017) An Unusual Cause of Anuria in a Young Patient with Hypertension. Saudi Journal of Kidney Diseases and Transplantation, 28, 1453-1454. https://doi.org/10.4103/1319-2442.220857

[10] Moriwaki, R., Noda, M., Yajima, M., Sharma, B.K. and Numano, F. (1997) Clinical Manifestations of Takayasu Arteritis in India and Japan-New Classification of Angiographic Findings. Angiology, 48, 369-379. https://doi.org/10.1177\%2F000331979704800501

[11] Baldwin, C., Mohammad, A.J., Cousins, C., Carette, S., Pagnoux, C. and Jayne, D. (2018) Long-Term Outcomes of Patients with Takayasu Arteritis and Renal Artery Involvement: A Cohort Study. Rheumatology Advances in Practice, 2, rky026. https://doi.org/10.1093/rap/rky026

[12] Fadouach, S., Abid-Allah, M., Mehadji, B.A., Tahiri, A. and Chraibi, N. (1998) Hypertension and Takayasu Disease. Report of 8 Cases. Annales de Cardiologie et D' angeiologie, 47, 155-159.

[13] Dardik, A., Ballermann, B.J. and Williams, G.M. (1998) Successful Delayed Bilateral Renal Revascularization during Active Phase of Takayasu's Arteritis. Journal of Vascular Surgery, 27, 552-554. https://doi.org/10.1016/S0741-5214(98)70332-5

[14] Wolak, T., Szendro, G., Golcman, L. and Paran, E. (2003) Malignant Hypertension as a Presenting Symptom of Takayasu Arteritis. Mayo Clinic Proceedings, 78, 231-236. https://doi.org/10.4065/78.2.231

[15] Zumrutdal, A., Tercan, F., Oguzkurt, L., Sezer, S. and Ozdemir, F.N. (2005) Report of a Case with Surprising Etiology of Renovascular Hypertension. Clinical Nephrology, 64, 444-447. https://doi.org/10.5414/CNP64444

[16] Galešić, K., Morović-Vergles, J., Živko, M., Račić, I., Vergles, D., Borković, Z. and Čikeš, N. (2005) Renovascular Hypertension Due to Takayasu Arteritis-A Case Report. Reumatizam, 52, 8-32.

[17] Moorthy, N., Prasad, B.R., Kumar, Y.L., Kumar, M.H. and Prathima, K.M. (2009) Malignant Hypertension, Dissection of Aorta, Cardiac Tamponade and Monoparesis-Unusual Presentation of Takayasu's Arteritis: Clinicopathological Correlation. International Journal of Cardiology, 133, e114-e117. https://doi.org/10.1016/j.ijcard.2007.12.042

[18] Borazan, A., Sevindik, O.G., Solmaz, D., Gulcu, A., Cavdar, C., Sifil, A., et al. (2009) A Rare Cause of Renovascular Hypertension: Takayasu Arteritis with Only Renal Artery Involvement. Renal Failure, 31, 327-331. https://doi.org/10.1080/08860220902780093 
[19] Hassani, M. (2016) Takayasu Arteritis Associated with Severe Renovascular Hypertension. Saudi Journal of Kidney Diseases and Transplantation, 27, 402-406. https://doi.org/10.4103/1319-2442.178581

[20] Horino, T., Ichii, O., Matsumoto, T. and Terada, Y. (2020) Takayasu Arteritis Presenting as Renovascular Hypertension. Journal of Clinical Rheumatology, 26, e94. https://doi.org/10.1097/RHU.0000000000000936

[21] Tsuchida, T., Yano, H., Raita, Y. and Kinjo, M. (2019) Nephrotic Range Proteinuria and Metabolic Alkalosis in Takayasu Arteritis. BMJ Case Reports CP, 12, Article ID: e228637. http://dx.doi.org/10.1136/bcr-2018-228637

[22] Vargas-Hitos, J.A., Jiménez-Jáimez, J., Molina Navarro, E., Salmerón Ruiz, A., López Milena, G. and Jiménez-Alonso, J. (2019) Takayasu Arteritis, Malignant Hypertension and Severe Left Ventricular Hypertrophy. International Journal of Rheumatic Diseases, 22, 951-955. https://doi.org/10.1111/1756-185X.13541 